\title{
Pyruvate sensitizes pancreatic tumors to hypoxia-activated prodrug $\mathrm{TH}-302$
}

Jonathan W Wojtkowiak ${ }^{1 *}$, Heather C Cornnell ${ }^{1}$, Shingo Matsumoto ${ }^{8}$, Keita Saito ${ }^{8}$, Yoichi Takakusagi $^{8}$, Prasanta Dutta ${ }^{1}$, Munju Kim³ ${ }^{3}$ Xiaomeng Zhang ${ }^{1}$, Rafael Leos ${ }^{5,7}$, Kate M Bailey ${ }^{1}$, Gary Martinez ${ }^{1}$, Mark C Lloyd ${ }^{4}$, Craig Weber ${ }^{5}$, James B Mitchell ${ }^{8}$, Ronald M Lynch ${ }^{5,6}$, Amanda F Baker ${ }^{5,7}$, Robert A Gatenby ${ }^{2,3}$, Katarzyna A Rejniak ${ }^{3,10}$, Charles Hart ${ }^{9}$, Murali C Krishna ${ }^{8}$ and Robert J Gillies ${ }^{1,2}$

\begin{abstract}
Background: Hypoxic niches in solid tumors harbor therapy-resistant cells. Hypoxia-activated prodrugs (HAPs) have been designed to overcome this resistance and, to date, have begun to show clinical efficacy. However, clinical HAPs activity could be improved. In this study, we sought to identify non-pharmacological methods to acutely exacerbate tumor hypoxia to increase TH-302 activity in pancreatic ductal adenocarcinoma (PDAC) tumor models.

Results: Three human PDAC cell lines with varying sensitivity to TH-302 (Hs766t > MiaPaCa-2 > SU.86.86) were used to establish PDAC xenograft models. PDAC cells were metabolically profiled in vitro and in vivo using the Seahorse XF system and hyperpolarized ${ }^{13} \mathrm{C}$ pyruvate MRI, respectively, in addition to quantitative immunohistochemistry. The effect of exogenous pyruvate on tumor oxygenation was determined using electroparamagnetic resonance (EPR) oxygen imaging. Hs766t and MiaPaCa-2 cells exhibited a glycolytic phenotype in comparison to TH-302 resistant line SU.86.86. Supporting this observation is a higher lactate/pyruvate ratio in Hs766t and MiaPaCa xenografts as observed during hyperpolarized pyruvate MRI studies in vivo. Coincidentally, response to exogenous pyruvate both in vitro (Seahorse oxygen consumption) and in vivo (EPR oxygen imaging) was greatest in Hs766t and MiaPaCa models, possibly due to a higher mitochondrial reserve capacity. Changes in oxygen consumption and in vivo hypoxic status to pyruvate were limited in the SU.86.86 model. Combination therapy of pyruvate plus TH-302 in vivo significantly decreased tumor growth and increased survival in the MiaPaCa model and improved survival in Hs766t tumors.
\end{abstract}

Conclusions: Using metabolic profiling, functional imaging, and computational modeling, we show improved TH-302 activity by transiently increasing tumor hypoxia metabolically with exogenous pyruvate. Additionally, this work identified a set of biomarkers that may be used clinically to predict which tumors will be most responsive to pyruvate $+\mathrm{TH}-302$ combination therapy. The results of this study support the concept that acute increases in tumor hypoxia can be beneficial for improving the clinical efficacy of HAPs and can positively impact the future treatment of PDAC and other cancers.

Keywords: Hypoxia, Hypoxia-activated prodrugs, TH-302, Tumor microenvironment, Metabolism, Pancreatic cancer, Functional imaging, Computational modeling

\section{Background}

Tumor hypoxia, a routinely observed phenotype, contributes to chemo- and radioresistance, causing major therapeutic concern [1]. Due to extensive hypoxia, considerable effort has been devoted to harness its therapeutic potential. To exploit hypoxia, a class of hypoxia-activated prodrugs

\footnotetext{
* Correspondence: Jonathan.Wojtkowiak@Moffitt.Org

'Department of Imaging and Metabolism, H. Lee Moffitt Cancer Center and Research Institute, Tampa, FL 33612, USA

Full list of author information is available at the end of the article
}

(HAPs) has been developed to selectively target tumor cells residing in hypoxic niches [2,3]. The most common class of HAPs is based on 2-nitroimidazoles, exemplified by $\mathrm{TH}-302$, which is activated by cytochrome $\mathrm{P} 450$ reductase and contains alkylating nitrogen mustard, Br-isophosphoramide, as a cytotoxic effector [4]. There are 12 clinical trials with $\mathrm{TH}-302$ underway in a variety of cancers (e.g., pancreatic, sarcoma, breast, melanoma) (Additional file 1: Table S1). 
The major predictor of $\mathrm{TH}-302$ activity is the extent of tumor hypoxia [5]. We therefore hypothesized that increasing tumor hypoxia could improve TH-302 activity. Experimental evidence show that the most effective approach to decrease tumor oxygenation $\left(\mathrm{pO}_{2}\right)$ is increasing the oxygen consumption rate (OCR) [6]. This was effective in RKO cells in which pharmacological inhibition of hypoxia-inducible factor 1-alpha (HIF-1 $\alpha$ ) with echinomycin increased OCR, decreased tumor $\mathrm{pO}_{2}$, and increased the activity of the HAP, tirapazamine [7]. While HIF- $1 \alpha$ inhibition was effective at increasing OCR, pharmacological inhibition may induce chronic hypoxia that could promote further adaptation and possibly increased HAP side effects. A separate study demonstrated that $\left[1-{ }^{13} \mathrm{C}\right]$ pyruvate infusion during hyperpolarized MRI studies substantially decreased tumor $\mathrm{pO}_{2}$ and increased hypoxia in squamous cell carcinoma (SCC) tumors [8]. The effect was transient, with peak hypoxia observed at $30 \mathrm{~min}$, returning to baseline within $5 \mathrm{~h}$. Pyruvate also stimulates OCR across a panel of breast cancer cell lines [9], consistent with historical data that shows increased OCR in response to pyruvate (or lactate) in neurons and cardiac myocytes [10-12]. Because pyruvate 1) transiently decreases tumor $\mathrm{pO}_{2}, 2$ ) decreases tumor $\mathrm{pO}_{2}$ metabolically rather than pharmacologically, and 3) produces no detectable side effects, we hypothesize that pyruvate would be a successful adjuvant to enhance TH-302 efficacy.

A consequence of a desmoplastic stroma is poor perfusion, particularly in pancreatic ductal adenocarcinoma (PDAC). PDAC tumors are profoundly hypoxic in comparison to normal pancreatic tissue [13] which is a negative prognostic factor [14]. TH-302 is highly effective in preclinical pancreatic models [15] and has been effective in phase I/II trials in combination with gemcitabine (GEM), increasing progression-free survival of late-stage cancers by 2 months [16]. These encouraging results justified a phase III trial, which is ongoing (NCT01746979).

In the present study, we utilized PDAC models to demonstrate the ability of exogenous pyruvate to stimulate oxygen consumption in vitro and transiently decrease tumor $\mathrm{pO}_{2}$ in vivo. The decrease in tumor $\mathrm{pO}_{2}$ correlated with improved TH-302 efficacy, demonstrated by significant tumor volume decreases and increased survival. We also identified potential imaging and histological biomarkers that can be used to predict responsive tumor types. These data support the concept that temporarily increasing tumor hypoxia can improve the efficacy of HAPs, including TH-302, against PDAC and other cancer types in the clinic.

\section{Methods}

Cell culture

SU.86.86 and Hs766t cells were obtained from Threshold Pharmaceuticals (Redwood City, CA, USA), and MiaPaCa-
2 cells were obtained from American Type Cell Collection (ATCC, Manassas, VA, USA). The cells were maintained in RPMI-1640 (Life Technologies) supplemented with $10 \%$ fetal bovine serum (FBS) (HyClone) (SU.86.86) and Dulbecco's modified Eagle's medium-F12 (DMEM-F12) (Life Technologies) supplemented with 10\% FBS (Hs766t and $\mathrm{MiaPaCa}-2)$. All three cell lines were resuscitated from low passage with all experiments carried out with cells of passage numbers less than 10 (SU.86.86) and 20 (Hs766t and MiaPaCa-2).

\section{Animal housing}

All animal experiments were carried out at the Moffitt Cancer Center and National Cancer Institute in compliance with the Guide for the Care and Use of Laboratory Animal Resources [17]. Experimental protocols conducted at Moffitt Cancer Center were approved by the Institutional Animal Care and Use Committee, University of South Florida. Experimental protocols performed at the National Cancer Institute were approved by the Institutional Animal Care and Use Committee, National Cancer Institute.

\section{Tumor development and treatment}

Hs766t, MiaPaCa-2, and SU.86.86 pancreatic cancer cells were suspended in Matrigel Matrix (BD Biosciences, Franklin Lakes, NJ, USA) and phosphate-buffered saline (PBS) (1:1). Female SCID mice (6 weeks) (Harlan Inc., Indianapolis, IN, USA) were inoculated with $5 \times$ $10^{6}-10 \times 10^{6}$ cells (matrigel/PBS) subcutaneously on the right flank to form xenograft tumors. Tumor volume was determined twice per week by caliper until the volume reached $300 \mathrm{~mm}^{3}$ upon which the mice were randomly placed into treatment groups $(N=10$ per group). Treatment groups consisted of saline control, TH$302(50 \mathrm{mg} / \mathrm{kg}$ (Figure 1) and $80 \mathrm{mg} / \mathrm{kg}$ ) and pyruvate (1.15 mmol/kg) (Sigma-Aldrich, St. Louis, MO, USA) plus TH-302. TH-302 was administered IP five times per week for 2 weeks. Pyruvate was administered IV $30 \mathrm{~min}$ prior to $\mathrm{TH}-302$ in combination studies. The tumor volumes were followed twice per week by caliper. Mice with tumors reaching 2,000 $\mathrm{mm}^{3}$ were removed from the study.

\section{Immunohistochemistry}

Surgically removed pancreatic xenografts were formalin fixed and paraffin embedded (FFPE). Untreated xenograft tissue cross sections were stained for carbonic anhydrase IX (anti-rabbit AB15086; Abcam, Cambridge, MA, USA), lactate dehydrogenase V (anti-rabbit AB9002; Abcam), monocarboxylate transporter 1 (anti-rabbit SC-50324; Santa Cruz Biotechnology, Santa Cruz, CA, USA), and CD31 (anti-rabbit AB28364; Abcam). PDAC xenografts treated with $\mathrm{TH}-302(50 \mathrm{mg} / \mathrm{kg})$ for $48 \mathrm{~h}$ were formalin fixed, 


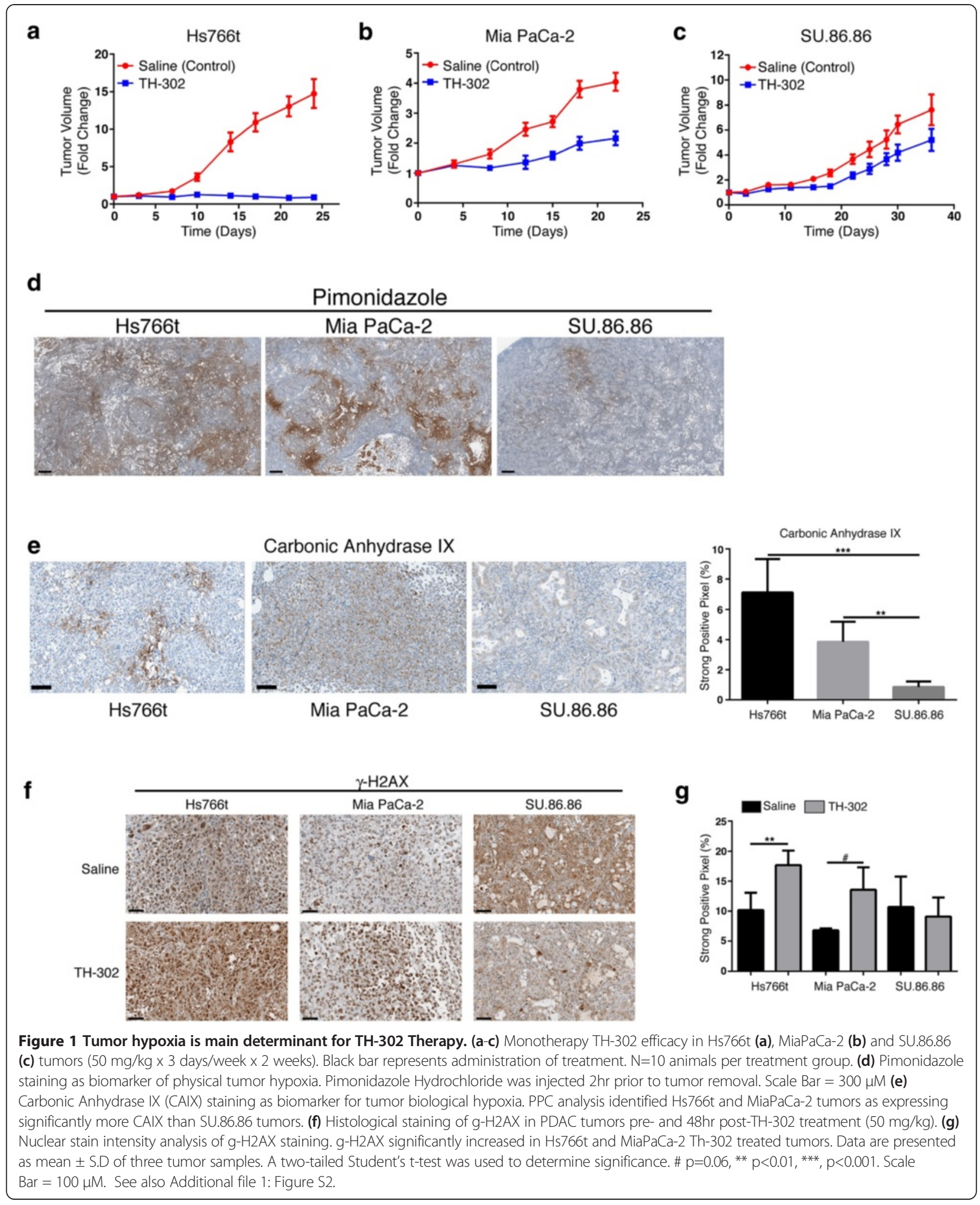

paraffin embedded, and stained for gamma-H2AX. Pimonidazole hydrochloride $(60 \mathrm{mg} / \mathrm{kg}$; Hypoxyprobe Inc.) was injected I.P. $1 \mathrm{~h}$ prior to tumor removal. Pimonidazole- positive tissue was detected using rabbit anti-sera against pimonidazole hydrochloride (2,627; Hypoxyprobe Inc.). A tissue microarray (TMA) containing FFPE human 


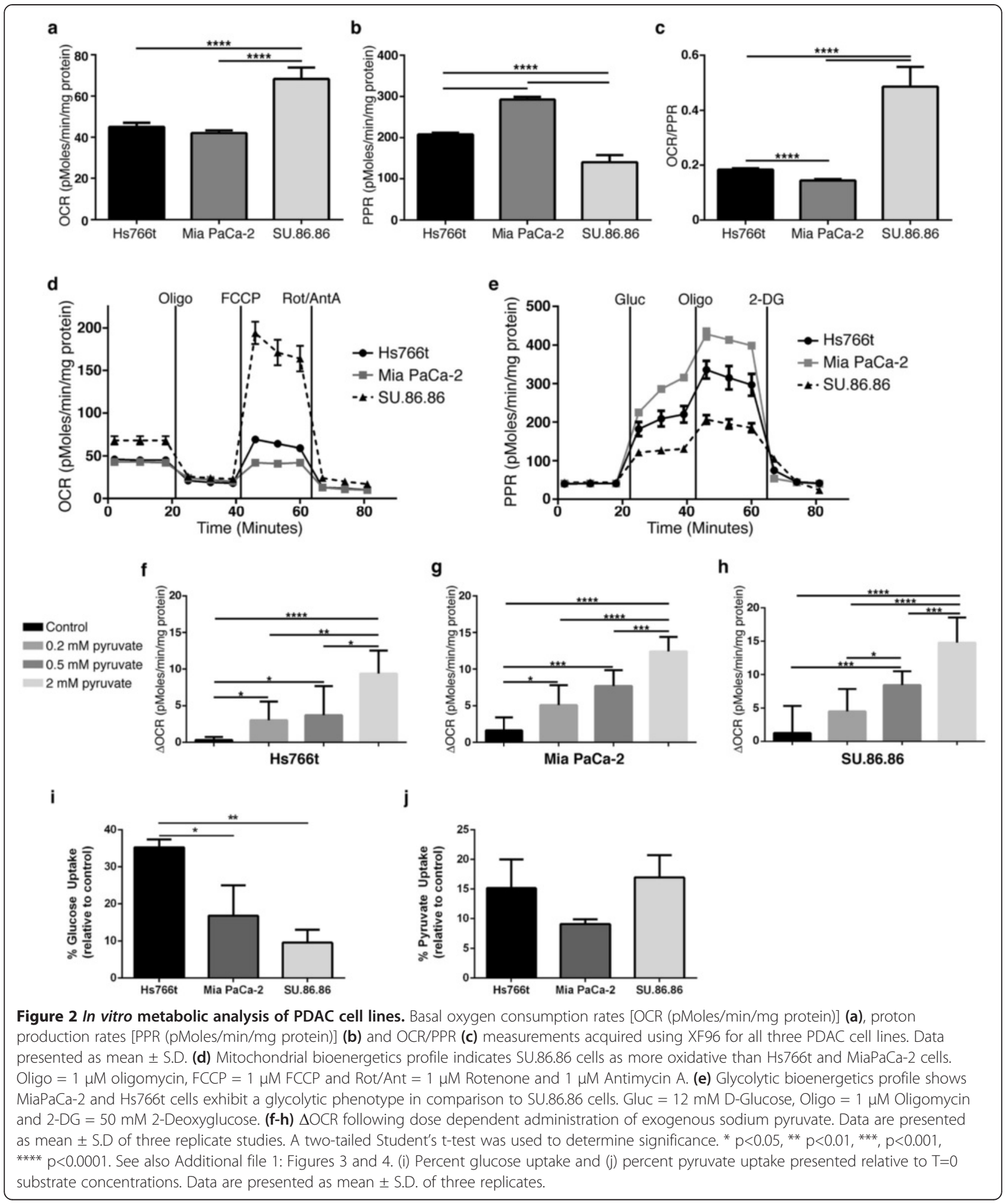

pancreatic adenocarcinoma specimen, acquired from Moffitt Cancer Center Total Cancer Care tissue database, was stained with antibodies against monocarboxylate transporter 1, monocarboxylate transporter 4 (anti-rabbit
SC-376140; Santa Cruz Biotechnology), and pyruvate kinase isoform M2 (anti-mouse \#3198; Cell Signaling Technology Inc., Danvers, MA, USA). The Ventana OmniMap anti-rabbit or anti-mouse secondary was used 
to detect primary antibodies. The detection system used was the Ventana ChromoMap Kit, and slides were then counterstained with hematoxylin. Histology stained slides were scanned using the Aperio ScanScope XT.

\section{Seahorse XF metabolic assays}

In vitro metabolic analysis using the XF96 (Seahorse Bioscience, Chicopee, MA, USA) was previously described [18]. Briefly, real-time OC (pMole/min) and proton production rates (PPR, $\mathrm{pMole} / \mathrm{min}$ ) are determined by measuring oxygen and free protons in the media over a monolayer of cells. All collected data are normalized to protein using a standard BCA protein assay. Data are presented as the normalized mean \pm standard deviation (S.D.)/mg protein.

\section{Mitochondrial stress test}

ATP-linked OCR and mitochondrial reserve capacity were determined following the sequential injections of oligomycin $(1 \mu \mathrm{M})$, carbonyl cyanide-p-trifluoromethoxyphenylhydrazone (FCCP) $(1 \mu \mathrm{M})$, and rotenone plus antimycin A $(1 \mu \mathrm{M})$. ATP-linked oxygen consumption is determined by the difference between basal OCR and OCR following addition of oligomycin. Mitochondrial reserve capacity is defined as the difference of basal OCR and OCR following the addition of FCCP.

\section{Glycolysis stress test}

Glycolysis and glycolytic capacity were determined following sequential injections of D-glucose (2 g/L), oligomycin $(1 \mu \mathrm{M})$, and 2-deoxyglucose $(100 \mathrm{mM})$. Initially, the cells are glucose starved for $2 \mathrm{~h}$ prior to experiment start. Glycolysis was defined as PPR following the addition of $\mathrm{D}$-glucose, and maximum glycolytic capacity was defined as PPR following the addition of oligomycin. PPR following treatment with 2-deoxyglucose is associated with non-glycolytic activity.

\section{Glucose and pyruvate uptake assay}

Glucose (K676) and pyruvate (K609) uptake from culture media was measured using colometric from BioVision Inc. The PDAC cells were seeded in standard growth media (see "Cell culture" section) and allowed to adhere overnight. At $T=0$, the cells were rinsed twice with PBS and provided DMEM supplemented with $5.5 \mathrm{mM}$ D-glucose, $1 \mathrm{mM}$ L-glutamine, $2 \mathrm{mM}$ sodium pyruvate, and $0.2 \%$ FBS. Following $12 \mathrm{~h}$ of culture time, the media were collected, centrifuged to remove cell debris, and immediately tested for glucose and pyruvate following BioVision assay kit protocols and compared to starting media conditions. Absorbance was quantified at $570 \mathrm{nM}$. Data are presented as percent substrate uptake (mean \pm S.D.) relative to $T=0$ culture media concentrations.

\section{EPR imaging}

A pulsed electroparamagnetic resonance (EPR) scanner with a parallel coil resonator tuned to $300 \mathrm{MHz}$ was used for oxygen imaging. After the mouse was placed in the resonator, triarylmethyl EPR oxygen tracer OX063 (1.125 $\mathrm{mmol} / \mathrm{kg}$ bolus followed by $0.12 \mathrm{mmol} / \mathrm{min}$ of continuous infusion) was administrated via tail veil cannula. Single-point imaging with a three-gradient set was used to generate $\mathrm{T}_{2}^{*}$ map, i.e., EPR line width map, which linearly correlates with local concentration of oxygen and allows pixelwise estimation of $\mathrm{pO}_{2}$. In total, four $\mathrm{pO}_{2}$ maps were obtained for each mouse before, 10, 30, and $60 \mathrm{~min}$ after IV injection of pyruvate $(1.15 \mathrm{mmol} / \mathrm{kg}$, bolus). After EPR study, the mice were transferred to a 7T MRI scanner (Bruker Bio-Spin MRI GmbH) for anatomic imaging.

\section{Mathematical model}

The previously developed mathematical model microPD [19], which couples the reaction-diffusion/convection equations with the fluid-structure interaction methods on spatially explicit tissue structure, has been calibrated to match the histology of MiaPaCa-2 mouse xenograft, the PK/PD properties of oxygen, and both inactive and active TH-302 (Additional file 1: Methods). The simulations were executed, visualized, and analyzed using a suite of in-house Matlab routines. Tissue histology was digitized using ImageJ software and in-house Matlab routines.

\section{Statistical analysis}

A two-tailed unpaired Student's $t$-test was used to determine statistical significance. The significance level was set at $p<0.05$.

\section{Results}

\section{Identifying baseline TH-302 sensitivity in human PDAC} tumor models

Three human PDAC cell lines (Hs766t, MiaPaCa-2, and SU.86.86) were used to evaluate the effect of exogenous pyruvate on tumor hypoxia and TH-302 efficacy. Subcutaneous tumors were shown to be histologically (H\&E) similar to pancreatic orthotopic tumors (Additional file 1: Figure S1). These were used to accommodate imaging modalities used to monitor pyruvate metabolism and tumor oxygenation, which cannot currently be made with orthotopic PDAC tumors. TH-302 monotherapy studies were carried out against each tumor type. The greatest anti-tumor effect occurred in Hs766t tumors. MiaPaCa-2 tumors had an intermediate response, and SU.86.86 tumors displayed no sensitivity to TH-302 (Figure 1a-c). As hypoxia is the main determinant for $\mathrm{TH}-302$ activity, immunohistochemistry (IHC) detecting pimonidazole and carbonic anhydrase IX, respectively, markers for physical [20] and biological [21] hypoxia, confirmed the greatest 
hypoxia in Hs766t tumors (Figure 1d,e). Hs766t cells also carry a nonsense mutation in FANCG [22] rendering them deficient in DNA repair and hence sensitizing them to alkylating agents, such as Br-ifosphoramide, the effector agent in TH-302. To confirm sensitivity to such agents, all three lines were treated with DNA cross-linking agent, mitomycin C (MMC), under normoxic conditions. Hs766t cells exhibited extreme sensitivity to low nanomolar concentrations of $\mathrm{MMC}\left(\mathrm{IC}_{50}=2.8 \mathrm{nM}\right)$ in comparison to MiaPaCa-2 $\left(\mathrm{IC}_{50}=15.1 \mathrm{nM}\right)$ and SU.86.86 $\left(\mathrm{IC}_{50}=29.7\right.$ nM) cells which carry no detectable DNA repair defects (Additional file 1: Figure S2a). Negligible TH-302 activity in SU.86.86 may be attributed to a well-oxygenated tumor environment as evidence by low CA9 expression and reduced sensitivity to DNA cross-linking agents. In vivo tumor doubling times also correlated with TH-302 sensitivity, as faster growing tumors (Hs766t) were more hypoxic and sensitive to TH-302 than slower growing tumors (SU.86.86), which we interpret as a higher propensity to outgrow existing blood vessels (Additional file 1: Figure S2b). Quantification of vascular density based on CD31 staining supports this observation as Hs766t $(p=0.0526)$ and MiaPaCa-2 $(p=0.0189)$ tumors had less vascular density than SU.86.86 tumors (Additional file 1: Figure S3). Th-302 toxicity was validated by quantification of $\gamma-\mathrm{H} 2 \mathrm{AX}$, a DNA damage response reporter [5] (Additional file 1: Figure S2d). $\gamma-\mathrm{H} 2 \mathrm{AX}$ increased in treated Hs766t $(p=0.004)$ and MiaPaCa-2 $(p=0.061)$ tumors with no change in SU.86.86 tumors $(p=0.669)$ (Figure 1f,g).

Pyruvate stimulates oxygen consumption in glycolytic cells The Seahorse XF96 was used to determine metabolic phenotypes (i.e., oxidative or glycolytic) for each cell line. The OCR of Hs766t (42 pMoles/min/mg) and MiaPaCa2 (40 pMoles/min/mg) cells were significantly lower than that of SU.86.86 (65 pMoles/min/mg) (Figure 2a). Mitochondrial stress tests indicated that SU.86.86 cells had greater ATP-linked OCR and mitochondrial reserve capacity than $\mathrm{Hs} 766 \mathrm{t}$ and $\mathrm{MiaPaCa}-2$ cells, implying that the SU.86.86 cells are more oxidative (Figure $2 \mathrm{~d}$ and Additional file 1: Figure S4a-c). Consistent with this interpretation, Hs766t and MiaPaCa-2 cells exhibited glycolytic phenotypes. Glucose-stimulated acid production (PPR) for Hs766t (179 pMoles/min/mg) and MiaPaCa-2 (275 $\mathrm{pMoles} / \mathrm{min} / \mathrm{mg}$ ) cells were higher than that for SU.86.86 (87 pMoles/min/mg) (Figure 2b). ATP-linked glycolysis and glycolytic capacity were also greater in Hs766t and MiaPaCa-2 cells (Figure 2e and Additional file 1: Figure S4d-f). Glucose consumption was quantified and shown to be greatest in Hs766t and least in SU.86.86 cells partially supporting the Seahorse metabolic data (Figure 2i). These metabolic data suggest that Hs766t and $\mathrm{MiaPaCa}-2$ cells are better suited to thrive under hypoxic conditions. Interestingly, SU.86.86 cells grew better under hypoxia than Hs766t and MiaPaCa-2 cells, even though the latter cell lines exhibited glycolytic phenotypes and formed more hypoxic tumors (Additional file 1: Figure S2c).

Pyruvate was administered to cells in increasing doses (0-2 $\mathrm{mM})$ with expectations that respiration would increase. $2 \mathrm{mM}$ pyruvate stimulated the largest OCR response (Figure 2f-h). Hs766t cells exhibited the largest relative increase and SU.86.86 cells the lowest, despite the fact that they had the largest oxidative reserve (Additional file 1: Figure S5e). No significant difference of pyruvate consumption was observed between cell lines, suggesting that intracellular pyruvate utilization may differ between cell lines (Figure 2j). PPR response to pyruvate was inversely related to OCR, presumably due to a reduction in net acid production (i.e., lactate derived from pyruvate rather than glucose), as well as a greater concentration of pyruvate entering the tricarboxylic acid cycle (TCA) cycle (Additional file 1: Figure S5a-d). Mitochondrial membrane potential increased following administration of 4 $\mathrm{mM}$ pyruvate in SU.86.86 cells as determined using tetramethylrhodamine ethyl ester (TMRE), confirming stimulation of the TCA cycle (Additional file 1: Figure S5f). These data indicate that cells with a glycolytic phenotype are more susceptible to pyruvate-stimulated OCR than oxidative SU.86.86 cells.

We performed ${ }^{13} \mathrm{C}$ magnetic resonance spectroscopy (MRS) of hyperpolarized $\left[1-{ }^{13} \mathrm{C}\right]$ pyruvate to validate the metabolic phenotypes in vivo. The pyruvate-to-lactate conversion in real-time is shown (Figure $3 a-f$ ), and the flux ratio (Lac/Pyr) was calculated from the area under the curve (AUC) for lactate and pyruvate versus time $(N=4)$. The Lac/Pyr flux ratio in Hs766t and MiaPaCa-2 tumors were significantly greater than that in SU.86.86 tumors (Figure 3g). Apparent rate constants $k_{\mathrm{P}}$ (Pyr/Lac) and $k_{\mathrm{L}}(\mathrm{Lac} / \mathrm{Pyr})$ were calculated using modified Bloch equations [23]. The $k_{\mathrm{P}} / k_{\mathrm{L}}$ ratio was significantly higher in Hs766t $(3.3 \pm 0.5)$ and MiaPaCa-2 $(3.4 \pm 0.4)$ than SU.86.86 $(2.7 \pm 0.4)$ tumors. The Lac/Pyr flux and $k_{\mathrm{P}} / k_{\mathrm{L}}$ ratios corresponded with the glycolytic phenotype of Hs766t and MiaPaCa-2 observed in vitro (Figure 2e and Additional file 1: Figure S4d-f). Hs766t and MiaPaCa-2 tumors also histologically exhibited higher lactate dehydrogenase A protein expression than SU.86.86 (Figure 3h,i).

\section{Pyruvate transiently decreases tumor oxygenation}

Electron paramagnetic resonance imaging (EPRI) was used to quantify changes in tumor $\mathrm{pO}_{2}$ following pyruvate administration [8]. Following anatomic MRI (left column $(\mathrm{T} 2 \mathrm{~W}))$ and prior to EPRI, the triarylmethyl radical (TAM) OX063 tracer was injected intravenously (IV) and baseline tumor $\mathrm{pO}_{2}$ determined (Figure $4 \mathrm{a}-\mathrm{c}$ ). Hs766t and MiaPaCa-2 tumors were hypoxic with a 


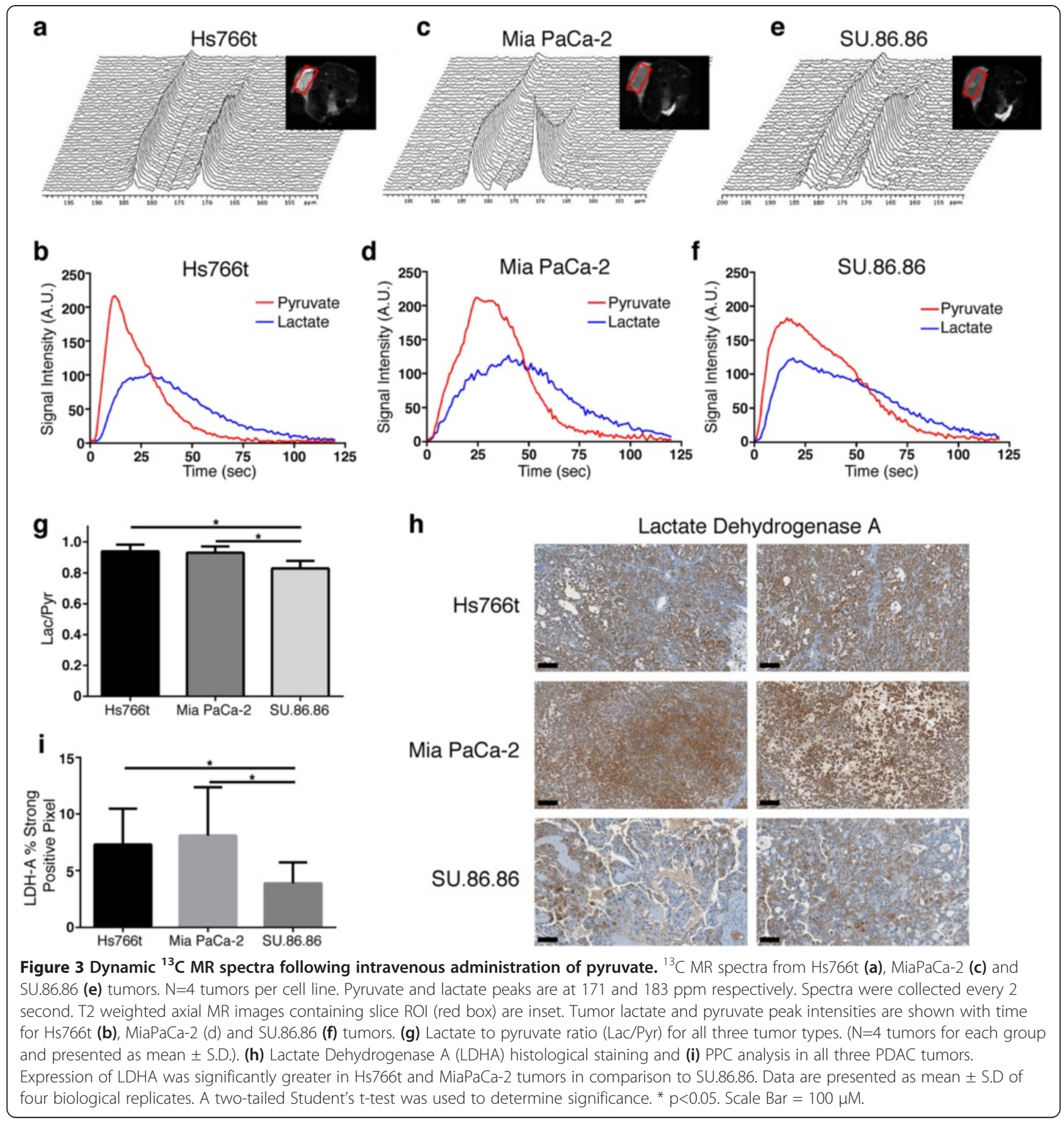

mean $\mathrm{pO}_{2}$ of $9.1 \pm 1.7$ and $11.1 \pm 2.2 \mathrm{mmHg}$, respectively (Figure $4 \mathrm{~d}$ ). Approximately $50 \%$ of the tumor volumes had a $\mathrm{pO}_{2}<10 \mathrm{mmHg}$ (Figure 4e), the $\mathrm{pO}_{2}$ at which TH-302 is activated [24]. The SU.86.86 tumors were less hypoxic with a mean tumor $\mathrm{pO}_{2}$ of $17.6 \pm 2.6 \mathrm{mmHg}$. Previously published data show that the $\mathrm{pO}_{2}$ of normal mouse muscle is $20.8 \pm 3.3 \mathrm{mmHg}$ [25]. Only $28 \%$ of SU.86.86 tumor volumes had a $\mathrm{pO}_{2}<10 \mathrm{mmHg}$. SU.86.86 consumed $\mathrm{O}_{2}$ at a higher rate in vitro, indicating that although there is no direct correlation between OCR and hypoxia in the steady state, it is likely modulated by the vascular oxygen supply. Hence, SU.86.86 tumors are better perfused, as observed by the higher AUC for pyruvate delivery (Figure 3f). Pyruvate $(1.15 \mathrm{mMol} / \mathrm{kg})$ was administered IV and tumor $\mathrm{pO}_{2}$ monitored and presented as mean tumor $\mathrm{pO}_{2}$ and hypoxic fraction (HF) of the entire tumor region. In SU.86.86 and Hs766t tumors, the greatest decrease in $\mathrm{pO}_{2}$ was observed 30 min post injection with $\mathrm{pO}_{2}$ recovering by $60 \mathrm{~min}$ (Figure $4 \mathrm{a}-\mathrm{d}$ ). In MiaPaCa-2 tumors, the $\mathrm{pO}_{2}$ continued to decline 


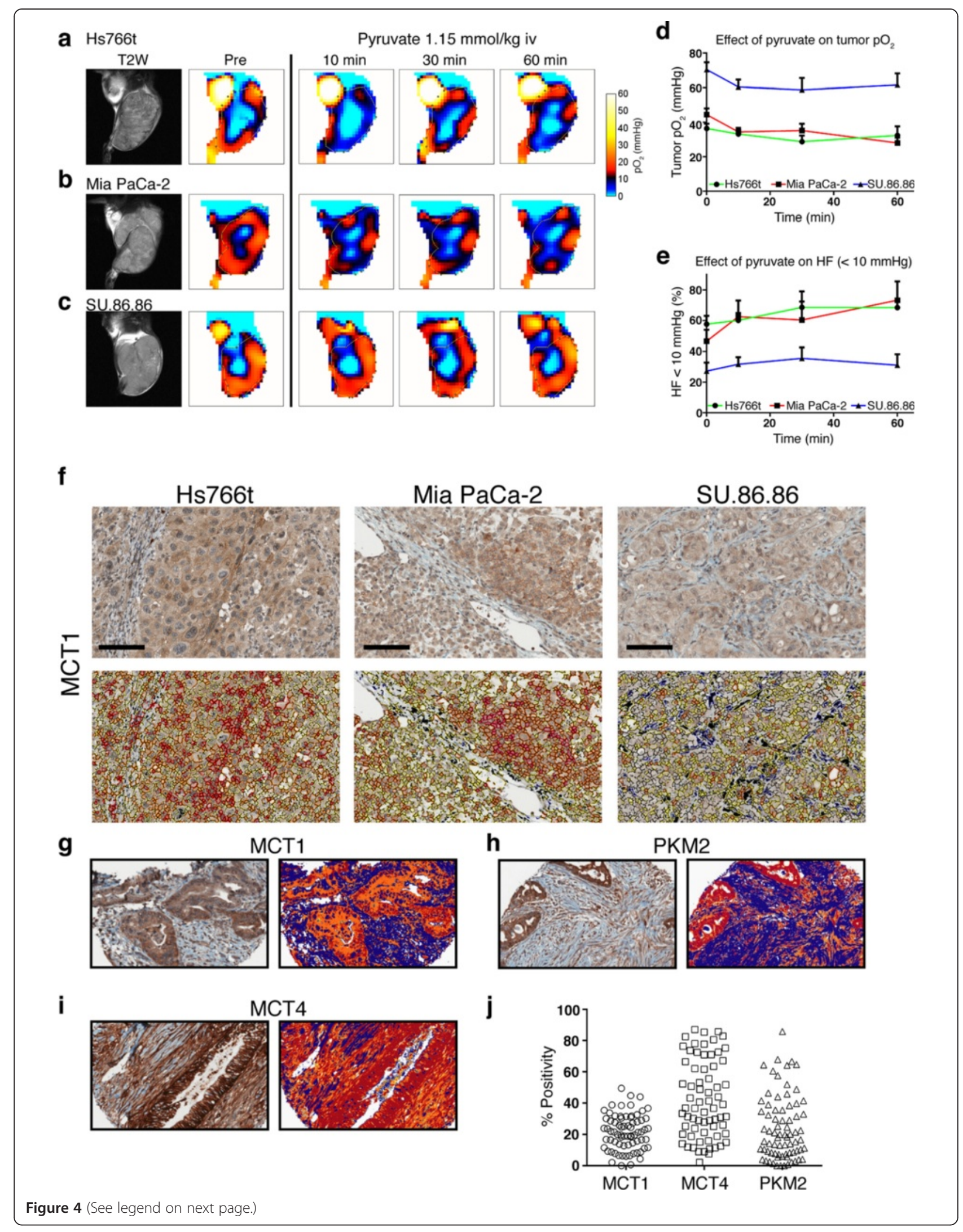


(See figure on previous page.)

Figure 4 EPR tumor oxygen imaging following administration of exogenous pyruvate. (a-c) EPR oxygen imaging of subcutaneous Hs766t (a), MiaPaCa-2 (b), and SU.86.86 (c) tumors pre- and post (10-60 min) IV pyruvate administration. Representative T2 weighted anatomical MR imaging and $\mathrm{pO}_{2}$ maps are provided $\mathrm{N}=4$ biological replicates per tumor type. (d) Temporal changes of mean $\mathrm{pO}_{2}$ and (e) percent hypoxic fraction $(<10 \mathrm{mmHg}$ ) of pyruvate-treated PDAC tumors. Data presented as mean \pm S.D. Proposed histological predictive biomarkers for pyruvate sensitivity. (f) Histological staining of monocarboxylate transporter (MCT) 1 expression in PDAC subcutaneous tumors. Corresponding images of positive pixel membrane staining are included. Human PDAC tissue microarray stained for MCT1 (g), MCT4 (h), and pyruvate kinase isoform M2 (i). (j) Distribution of percent positivity across well-differentiated human PDAC tissue cores. Scale bar = $100 \mu M$. See also Additional file 1: Figure S5.

throughout the study with no indication of recovery. The HF (pixels $<10 \mathrm{mmHg}$ ) significantly increased in both MiaPaCa-2 (22\%) and Hs766t (12\%) tumors. SU.86.86 baseline HF (28\%) was considerably lower than the other two tumor types, increasing only 7\%, 30 min after administering pyruvate, and returning to near baseline by 60 min (Figure 4e). Representative frequency histograms of tumor $\mathrm{pO}_{2}$ demonstrated a leftward shift after pyruvate administration in Hs766t and MiaPaCa-2 tumors with an increased percentage of pixels $<10 \mathrm{mmHg}$ (Figure $4 \mathrm{f}-\mathrm{h}$ and Additional file 1: Figure S6a-f). The sensitivity of Hs766t and MiaPaCa-2 tumors to pyruvate in vivo corroborate the in vitro metabolic profiling studies (cf. Figure 2). Additionally, low tumor $\mathrm{pO}_{2}$ and high HF of Hs766t and MiaPaCa-2 tumors at baseline may contribute to their native sensitivity to TH-302 monotherapy (Figure 1).

To investigate underlying mechanisms for pyruvate sensitivity in vivo, untreated pancreatic tumors were stained for monocarboxylate transporter 1 (MCT-1), a high affinity Pyr/Lac membrane transporter $(\mathrm{Km}=0.7 \mathrm{mM} / 3.5 \mathrm{mM})$ [26] (Figure 4f). Expression was higher in Hs766t and MiaPaCa-2 tumors, compared to SU.86.86. MCT-1 was quantified per cell, shown as a heat map in the lower panels of Figure 4f. Expression was heterogeneous with relatively small populations of high MCT-1 expressing cells (red) surrounded by low expressing cells (yellow or blue). To determine if this histological observation was clinically relevant, we stained a human PDAC tissue microarray for MCT-1 and additional glycolytic markers, PKM2 and MCT-4 [27] (Figure $4 \mathrm{~g}-\mathrm{j}$ ). All markers exhibited strong staining patterns in poorly differentiated ductal epithelial cells, as well as in stromal fibroblasts, indicating that stromal cells have altered metabolic activity, similar to the cancer cells. Stroma exhibiting such staining patterns may also contribute to hypoxic exacerbation by increasing respiration in their compartment, and this is under active investigation.
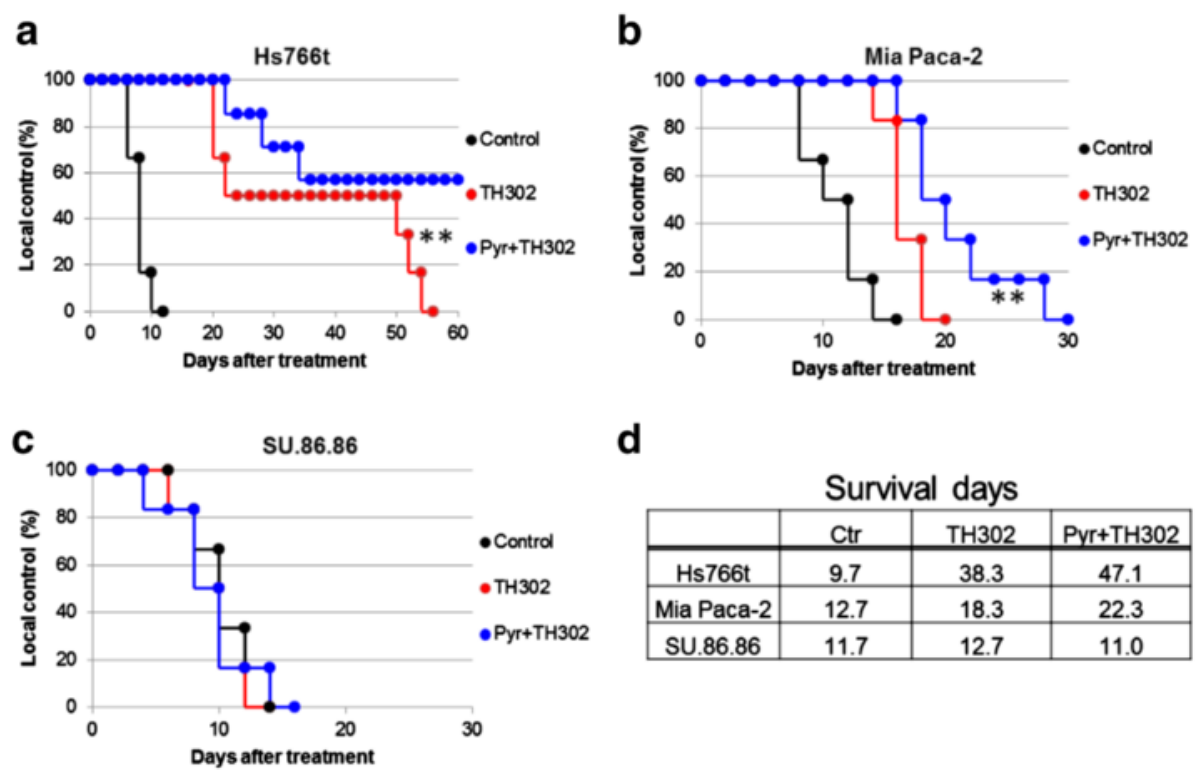

d

\begin{tabular}{|c|c|c|c|}
\multicolumn{4}{c|}{ Survival days } \\
\hline & Ctr & TH302 & Pyr+TH302 \\
\hline Hs766t & 9.7 & 38.3 & 47.1 \\
\hline Mia Paca-2 & 12.7 & 18.3 & 22.3 \\
\hline SU.86.86 & 11.7 & 12.7 & 11.0 \\
\hline
\end{tabular}

Figure 5 In vivo effect of pyruvate pretreatment on improving TH-302 efficacy. Percent local tumor control of Hs766t (a), MiaPaCa-2 (b), and SU.86.86 (c) tumors were treated with saline, TH-302 alone ( $80 \mathrm{mg} / \mathrm{kg} \times 5$ days I.P.) or TH-302 following a 30 min pretreatment with exogenous pyruvate ( $1.15 \mathrm{mMol} / \mathrm{kg}$ pyruvate IV $30 \mathrm{~min}$ prior to $80 \mathrm{mg} / \mathrm{kg}$ TH-302 I.P. $\times 5$ days). Response was measured as percentage of surviving animals as mice are removed from study when tumors reach $2,000 \mathrm{~mm}^{3}$. Pyruvate pretreatment significantly improved local control of Hs766t $(p<0.00225)$ and MiaPaCa-2 tumors with no measurable effect against SU.86.86. (d) Mean survival (days) of mice with pancreatic tumors treated with TH-302 and TH302 in combination with pyruvate pre-treatment. $N=10$ mice per treatment group. A two-tailed Student's $t$-test was used to determine significance. ${ }^{* *} p<0.01$. 


\section{Effect of pyruvate on TH-302 efficacy}

Since TH-302 is activated below $10 \mathrm{mmHg}$, we predicted that pyruvate pretreatment $(30 \mathrm{~min}$ ) would enhance $\mathrm{TH}$ 302 efficacy in Hs766t and MiaPaCa-2 tumors with little to no anticipated benefit in SU.86.86 tumors, as pyruvate had little effect on hypoxia in this model. PDAC tumors were established, and the mice were randomized into three treatment groups: 1) saline control, 2) TH-302 alone, and 3) pyruvate $+\mathrm{TH}-302$ (pyruvate 30 min prior to TH-302). Data are presented as percent local control of tumor growth. SU.86.86 tumors remained resistant to TH-302 alone with no benefit in survival when combined with pyruvate (Figure $5 \mathrm{c}, \mathrm{d}$ ). Pyruvate had no effect on the initial Hs766t TH-302 tumor response, as they were already maximally responsive (Figure 1a). However, pyruvate pretreatment significantly improved overall Hs766t survival (Figure $5 \mathrm{a}, \mathrm{d}$ ) with $50 \%$ of the animals surviving beyond 54 days, at which time all animals in the other groups had expired. MiaPaCa-2 tumors exhibited the greatest decrease in tumor $\mathrm{pO}_{2}$ by exogenous pyruvate (Figure 4). These tumors received the greatest benefit from pyruvate pretreatment with a significant delay in tumor growth compared to both saline control and TH-302 monotherapy groups. Combining pyruvate with TH-302 increased the time to reach $2,000 \mathrm{~mm}^{3}$ compared to tumors treated with TH-302 monotherapy $(p<0.05)$ and overall survival significantly increased by 9.7 days $(p<0.005$ in the pyruvate plus TH-302 treatment group compared to saline control) and by 4.7 days, compared to TH-302 monotherapy (log rank $p<0.05$ ) (Figure $5 \mathrm{~b}, \mathrm{~d}$ ).

\section{MicroPD model}

The pharmacokinetics of combination therapies are complex. In order to attain quantitative understanding, a micro-scale spatially explicit reaction-diffusion/convection pharmacodynamics (microPD) model [19] parameterized with experimental measurements from $\mathrm{MiaPaCa}-2$ tumors was developed to (refer to Additional file 1: Methods for model parameters) 1) explore TH-302 tissue penetration, 2) monitor TH-302 activation and diffusion of the released effector \pm pyruvate, and 3 ) monitor tissue hypoxia \pm pyruvate. For these analyses, tissue was digitized (Figure 6a) to spatially inform simulations (Figure $6 \mathrm{~b}-\mathrm{d}$ ) to show diffusive distributions of oxygen, pyruvate, inactive $\mathrm{TH}-302$, and active $\mathrm{TH}-302$ at varying distances from vasculature (Figure $6 \mathrm{~b}-\mathrm{d}$ ). The digitization contained individual cells, as well as interstitial parenchyma, that have the ability to consume oxygen, import pyruvate, and die when exposed to active TH-302. Baseline simulations showed that the hypoxia border $(<10 \mathrm{mmHg})$ is, on average, $110 \mu \mathrm{m}$ from the vasculature (Figure $6 \mathrm{~b}$ ). The measured diffusion distance of oxygen in tissue is between 100 and $200 \mu \mathrm{m}$ [28]. With the addition of pyruvate (Figure 6c), cells closest to vasculature consumed more oxygen, reducing oxygen diffusion (hypoxia border $=76 \mu \mathrm{m}$ ) leading to a $30 \%$ hypoxia increase (Figure 2c). The leftward shift of the hypoxia border is paramount as the distance by which inactive TH-302 must diffuse prior to activation is shortened, increasing the region exposed to the effector threefold. TH-302 treatment in the presence of pyruvate increased cell death (black dots) by $88 \%$ when compared to TH-302 alone (Figure 6d). An unexpected result of these simulations was a mechanism for tissue re-oxygenation following combination treatment. A rightward shift ( $T=30$ post $\mathrm{TH}-302)$ of the hypoxia border from 76 to $104 \mu \mathrm{m}$ occurs as oxygen consumption decreases as cell death increases. These simulations quantitatively illustrate how exogenous pyruvate may increase local hypoxia and thereby improve TH-302 efficacy.

\section{Discussion}

Because hypoxic tumors are poorly prognostic and resistant to chemo- and radiotherapies, there has been intense interest in agents that will target hypoxic volumes. HAPs are a new class of anti-cancer therapeutics, and TH-302 is a leading candidate in clinical trials. The pharmacodynamic effects of these agents are complex and depend on vascular delivery, tissue penetration-to-hypoxic volumes, activation, and bystander killing. This is made even more complex by the short plasma half-lives of these agents. We thus contend that increased efficacy can be achieved through transient exacerbation of hypoxia in combination with advanced pharmacodynamics modeling. We have shown that exogenous pyruvate transiently exacerbates tumor hypoxia by increasing the rate of cellular OCR leading to improved TH-302 anti-tumor efficacy. This work was performed in three PDAC models: Hs766t, MiaPaCa2, and SU.86.86 cells that have high, moderate, and low sensitivity to TH-302 monotherapy, respectively. Molecular and metabolic characterizations of these cells and tumors were consistent with their responses, as were their responses to pyruvate in vitro and in vivo. As predicted, pyruvate pretreatment caused a significant therapeutic benefit to the intermediately sensitive MiaPaCa-2 tumors exclusively. However, there were dramatic increases in survival for Hs766t tumors, which are highly sensitive to TH-302 monotherapy. Nonetheless, in contrast to the strong in vitro and in vivo effects of pyruvate on OCR and oxygen levels, the therapeutic benefits of combining pyruvate with HAP therapy, albeit significant, were only modest. Hence, we propose that additional optimization of timing and dose, using in silico models, can lead iteratively to improved therapeutic outcome of this novel combination therapy.

While the effect of exogenous pyruvate on metabolism has only recently been explored in the context of cancer 


\section{a}

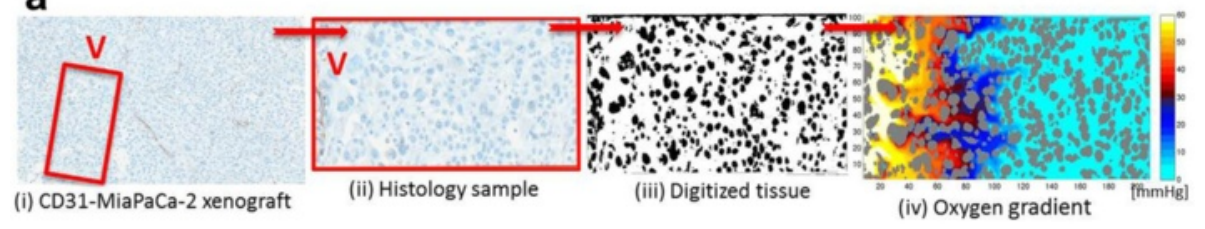

b

\section{Before treatment}

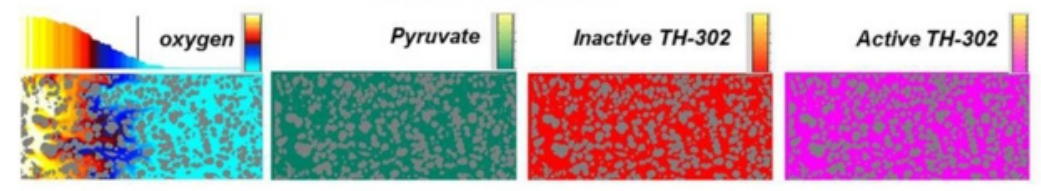

C
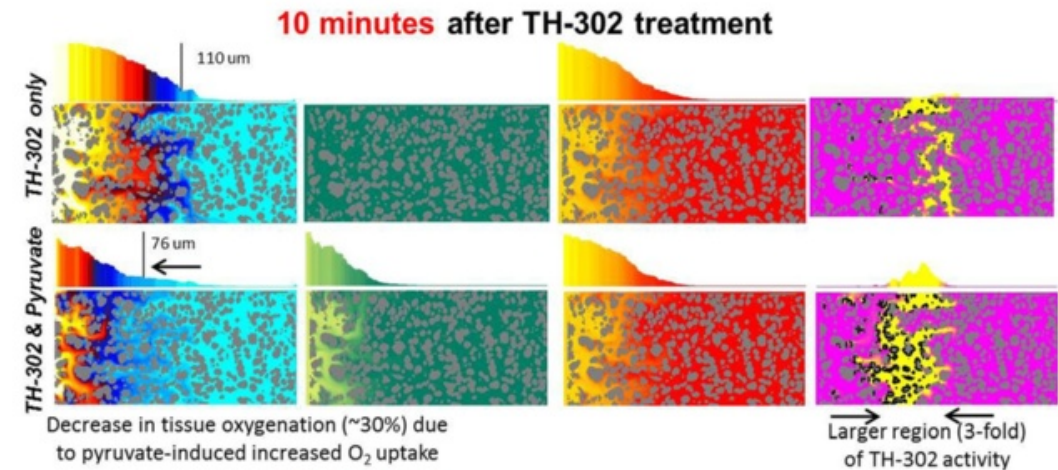

30 minutes after TH-302 treatment

d
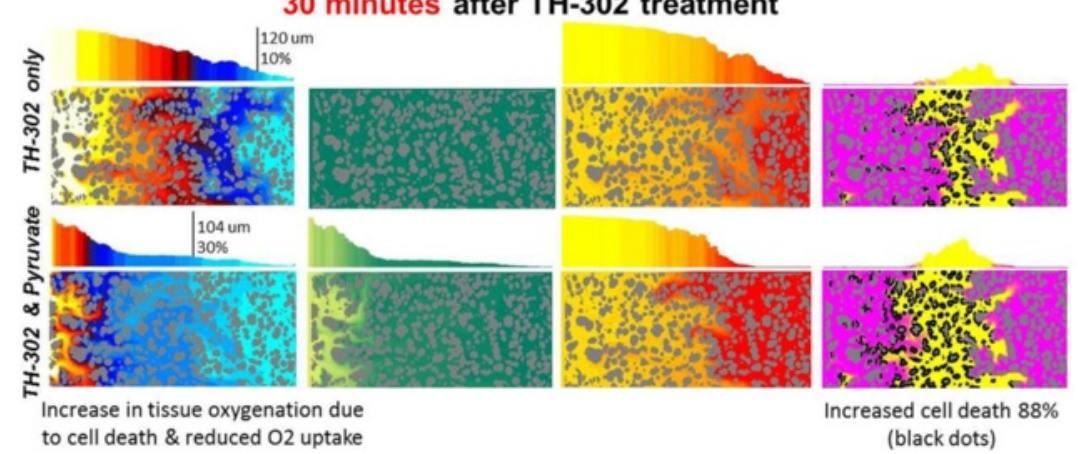

Increased cell death $88 \%$

(black dots)

Figure 6 Oxygen and active TH-302 distribution in simulated MiaPaCa-2 tissue extracellular space. (a) A ROI of MiaPaCa-2 xenograft tissue stained with CD-31 (i) was selected (ii) for segmentation (iii), and used as a domain for in-silico simulations of the interstitial transport of oxygen (IV). (b,c) Temporal and spatial distributions of oxygen (1st column), pyruvate (2nd column), inactive TH-302 (3rd column) and active TH-302 (4th column). The bar graphs show averaged amounts of the compound across the tissue slice. The vertical lines in oxygen bar graphs show the border between the normoxic (left) and hypoxic (right) regions of the tissue. Black dots in the active TH-302 tissue graph represent dead cells. (b) Initial distribution of oxygen before the treatment is applied. Pyruvate, inactive and active TH-302 are all absent. (c) Distributions of chemical compounds 10 min after applying TH-302. In the bottom-row simulation pyruvate was applied 30 min before TH-302 (second column) which resulted in decreased $\mathrm{pO} 2$ due to increased oxygen uptake by the cells exposed to pyruvate (first column), and in expanded region of TH-302 activation (last column). (d) Distributions of chemical compounds 30 min after applying TH-302. In the bottom-row simulation, pyruvate was applied 30 min before TH-302 (second column). The region of TH-302 activation in two-fold larger (yellow region in the last column), and the number of death cells is $88 \%$ larger (black dots in the last column) when compared to simulation with TH-302 only.

[8,9], pyruvate has been thoroughly investigated and identified in neuronal models as the predominant mitochondrial substrate [10]. We therefore hypothesized that reduced $\mathrm{pO}_{2}$ in pancreatic tumors by exogenous pyruvate was a result of elevated OCR. However, this does not explain why exogenous pyruvate alone would stimulate oxygen consumption in cancer cells. MiaPaCa-2 and Hs766t are glycolytic, implying that the pyruvate flux should be substantial and not limiting for mitochondrial consumption. However, the OCRs of these two tumor types were the most strongly affected by pyruvate. One possible explanation is that PKM2, the enzyme that catalyzes the dephosphorylation of phosphoenolpyruvate to pyruvate, is located adjacent to the plasma membrane, whereas the mitochondria are peri-nuclear $[29,30]$. The rate of pyruvate production is regulated by PKM2 and 
expression is consistently observed to be upregulated in transformed cells exhibiting glycolytic phenotypes. Since enzymes of the lower half of glycolysis are localized near the plasma membrane, we hypothesize that PKM2 is closely associated spatially with these enzymes, making the fate of pyruvate a readily available substrate for lactate dehydrogenase, rather than transport into the mitochondria [29]. Hence, PKM2-produced pyruvate must traverse several microns of intracellular space, with diffusion gradients and intervening bioconversions, before it can be used to fuel respiration. With these distances, reaction-diffusion models predict that the local concentration of pyruvate at PKM2 would have to be at least 100 times higher than the $\mathrm{Km}$ for mitochondrial consumption for the mitochondrial pyruvate dehydrogenase to become saturated [31].

In contrast, exogenous pyruvate may have a different metabolic fate than pyruvate generated via glycolysis. Following uptake, the process of converting pyruvate to lactate continues as shown by hyperpolarized ${ }^{13} \mathrm{C}$ pyruvate MR studies. However, since exogenous pyruvate can bypass PKM2, the available pyruvate concentration for transport into the mitochondria increases, stimulating respiration. Since pyruvate is delivered as a single bolus, exogenous pyruvate is quickly metabolized and the effect on respiration only transient; hence, the approximately 30 -min decrease of tumor $\mathrm{pO}_{2}$ in our pancreatic tumor model (Figure 4).

Finally, chronic hypoxia has been shown to select for cells that are resistant to apoptosis [32,33]. Hence, there may be concern that exacerbating hypoxia may lead to enhanced progression or therapy resistance. In contrast to previous studies that induced hypoxia pharmacologically over many days [7], the current metabolic approach lasts $<1 \mathrm{~h}$, which is consistent with the blood half-life of $\mathrm{TH}-302$. We contend that acute changes in $\mathrm{pO}_{2}$ will not lead to enhanced proliferation or resistance, but this will have to be investigated. Additionally, as pyruvate has also been shown to increase OCR in neurons, glia, and cardiac muscle [10-12], it is possible that this treatment may lead to hypoxia in normal tissues and increase $\mathrm{TH}$ 302 off-target effects. No increased toxicity of TH-302 was observed in our studies, and we reason that active maintenance of vessel tone in normal tissues, in contrast to tumors, exerts a protective effect by increasing blood flow to volumes with higher respiration. Nonetheless, further studies are warranted to investigate potential synergism between compounds that exacerbate tumor hypoxia and hypoxia-activated prodrugs.

\section{Conclusions}

PDAC has one of the worst prognoses of all cancers due to limited efficacy of current therapies. The hypoxia-activated prodrug, TH-302, has shown promise clinically but advances are needed to improve its efficacy against late stage pancreatic cancer. This study supports the concept that temporarily increasing tumor hypoxia with pyruvate can improve the efficacy of HAPs, including TH-302, against PDAC and other cancer types in the clinic. While the effect of pyruvate on TH-302 activity in vivo was significant, future studies are necessary to optimize timing and dose, using in silico models, iteratively leading to improved therapeutic outcome of this novel combination therapy.

\section{Additional file}

Additional file 1: Supplementary table and figures. This document contains additional supplemental data and extended methods section for the mathematical model. In total, one table and five supplemental figures are included.

\section{Competing interests}

$\mathrm{CH}$ is an employee and shareholder of Threshold Pharmaceuticals.

\section{Authors' contributions}

RJG initiated the project. JWW and RJG wrote the paper. JWW, RJG, RAG, JBM, SM, HCC, and MCK designed the experiments. JWW, HCC, SM, KS, YT, $P D, M C L, C W, X Z, R L, K M B, M K$ and KAR performed the experiments. JWW HCC, SM, KS, YT, PD, MCL, XZ, RL, KMB, KAR, GM, RML, AFB, and RJG analyzed the data. $\mathrm{CH}$ provided important reagents. All authors read and approved the final manuscript.

\section{Acknowledgements}

This work is supported by U.S. National Cancer Institute grants R01CA077575, R01CA125627, and U54CA143970 (RJG)

\section{Author details}

${ }^{1}$ Department of Imaging and Metabolism, H. Lee Moffitt Cancer Center and Research Institute, Tampa, FL 33612, USA. ²Department of Radiology, H. Lee Moffitt Cancer Center and Research Institute, Tampa, FL 33612, USA

${ }^{3}$ Department of Integrated Mathematical Oncology, H. Lee Moffitt Cancer Center and Research Institute, Tampa, FL 33612, USA. ${ }^{4}$ Analytic Microscopy Core Facility, H. Lee Moffitt Cancer Center and Research Institute, Tampa, FL 33612, USA. ${ }^{5}$ Arizona Cancer Center, College of Medicine, University of Arizona, Tucson, AZ 85724, USA. 'Department of Physiology, College of Medicine, University of Arizona, Tucson, AZ 85724, USA. ${ }^{7}$ Hematology/ Oncology Section, College of Medicine, University of Arizona, Tucson, AZ 85724, USA. ${ }^{8}$ Center for Cancer Research, National Cancer Institute, Bethesda, MD 20892, USA. 'Threshold Pharmaceutical, San Francisco, CA 94080, USA

${ }^{10}$ Department of Oncologic Sciences, University of South Florida, Tampa, FL 33612, USA.

Received: 21 August 2014 Accepted: 24 November 2014 Published online: 29 January 2015

\section{References}

1. Overgaard J: Hypoxic radiosensitization: adored and ignored. J Clin Oncol 2007, 25:4066-4074.

2. Denny WA: Hypoxia-activated prodrugs in cancer therapy: progress to the clinic. Future Oncol 2010, 6:419-428.

3. Wilson WR, Hay MP: Targeting hypoxia in cancer therapy. Nat Rev Cancer 2011, 11:393-410.

4. Meng F, Evans JW, Bhupathi D, Banica M, Lan L, Lorente G, Duan JX, Cai X Mowday AM, Guise CP, Maroz A, Anderson RF, Patterson AV, Stachelek GC, Glazer PM, Matteucci MD, Hart CP: Molecular and cellular pharmacology of the hypoxia-activated prodrug TH-302. Mol Cancer Ther 2012, 11:740-751.

5. Sun JD, Liu Q, Wang J, Ahluwalia D, Ferraro D, Wang Y, Duan JX, Ammons WS, Curd JG, Matteucci MD, Hart CP: Selective tumor hypoxia targeting by 
hypoxia-activated prodrug $\mathrm{TH}-302$ inhibits tumor growth in preclinical models of cancer. Clin Cancer Res 2012, 18:758-770.

6. Secomb TW, Hsu R, Ong ET, Gross JF, Dewhirst MW: Analysis of the effects of oxygen supply and demand on hypoxic fraction in tumors. Acta Oncol 1995, 34:313-316.

7. Cairns RA, Papandreou I, Sutphin PD, Denko NC: Metabolic targeting of hypoxia and HIF1 in solid tumors can enhance cytotoxic chemotherapy. Proc Natl Acad Sci U S A 2007, 104:9445-9450.

8. Saito K, Matsumoto S, Devasahayam N, Subramanian S, Munasinghe JP, Morris HD, Lizak MJ, Ardenkjaer-Larsen JH, Mitchell JB, Krishna MC Transient decrease in tumor oxygenation after intravenous administration of pyruvate. Magn Reson Med 2012, 67:801-807.

9. Diers AR, Broniowska KA, Chang CF, Hogg N: Pyruvate fuels mitochondrial respiration and proliferation of breast cancer cells: effect of monocarboxylate transporter inhibition. Biochem J 2012, 444:561-571.

10. Kauppinen RA, Nicholls DG: Synaptosomal bioenergetics. The role of glycolysis, pyruvate oxidation and responses to hypoglycaemia. Eur J Biochem 1986, 158:159-165.

11. Korzeniewski B, Harper ME, Brand MD: Proportional activation coefficients during stimulation of oxidative phosphorylation by lactate and pyruvate or by vasopressin. Biochim Biophys Acta 1995, 1229:315-322.

12. Schaffer WT, Olson MS: The regulation of pyruvate oxidation during membrane depolarization of rat brain synaptosomes. Biochem J 1980, 192:741-751.

13. Koong AC, Mehta VK, Le QT, Fisher GA, Terris DJ, Brown JM, Bastidas AJ, Vierra M: Pancreatic tumors show high levels of hypoxia. Int J Radiat Oncol Biol Phys 2000, 48:919-922.

14. Chang Q, Jurisica I, Do T, Hedley DW: Hypoxia predicts aggressive growth and spontaneous metastasis formation from orthotopically grown primary xenografts of human pancreatic cancer. Cancer Res 2011, 71:3110-3120.

15. Liu Q, Sun JD, Wang J, Ahluwalia D, Baker AF, Cranmer LD, Ferraro D, Wang Y, Duan JX, Ammons WS, Curd JG, Matteucci MD, Hart CP: TH-302, a hypoxiaactivated prodrug with broad in vivo preclinical combination therapy efficacy: optimization of dosing regimens and schedules. Cancer Chemother Pharmacol 2012, 69:1487-1498.

16. Borad MJ, Reddy S, Uronis H, Sigal DS, Cohn AL, Schelman WR, Stephenson J, Chiorean EG, Rosen PJ, Ulrich B, Dragovich T, Del Prete S, Rarick M, Eng C, Kroll S, Ryan D: Randomized phase Il study of the efficacy and safety of gemcitabine + TH-302 (G+T) vs gemcitabine (G) alone in previously untreated patients with advanced pancreatic cancer. Proceedings of the 103rd Annual Meeting of the American Association for Cancer Research 2012:LB-121.

17. Institute of Laboratory Animal Resources (U.S.): Guide for the care and use of laboratory animals. 7th edition. Washington, D.C: National Academy Press; 1996.

18. Ibrahim-Hashim A, Wojtkowiak JW, de Lourdes Coelho Ribeiro M, Estrella V, Bailey KM, Cornnell HH, Gatenby RA, Gillies RJ: Free Base Lysine Increases Survival and Reduces Metastasis in Prostate Cancer Model. J Cancer Sci Ther 2011, Suppl 1(4):JCST-S1-004.

19. Rejniak KA, Estrella V, Chen T, Cohen AS, Lloyd MC, Morse DL: The role of tumor tissue architecture in treatment penetration and efficacy: an integrative study. Front Oncol 2013, 3:111.

20. Varia MA, Calkins-Adams DP, Rinker LH, Kennedy AS, Novotny DB, Fowler WC $\mathrm{Jr}$, Raleigh JA: Pimonidazole: a novel hypoxia marker for complementary study of tumor hypoxia and cell proliferation in cervical carcinoma. Gynecol Oncol 1998, 71:270-277.

21. Wykoff CC, Beasley NJ, Watson PH, Turner KJ, Pastorek J, Sibtain A, Wilson GD, Turley H, Talks KL, Maxwell PH, Pugh CW, Ratcliffe PJ, Harris AL: Hypoxiainducible expression of tumor-associated carbonic anhydrases. Cancer Res 2000, 60:7075-7083.

22. van der Heijden MS, Brody JR, Gallmeier E, Cunningham SC, Dezentje DA, Shen D, Hruban RH, Kern SE: Functional defects in the fanconi anemia pathway in pancreatic cancer cells. Am J Pathol 2004, 165:651-657.

23. Dutta P, Le A, Vander Jagt DL, Tsukamoto T, Martinez GV, Dang CV, Gillies RJ: Evaluation of LDH-A and glutaminase inhibition in vivo by hyperpolarized 13C-pyruvate magnetic resonance spectroscopy of tumors. Cancer Res 2013, 73:4190-4195.

24. Arteel GE, Thurman RG, Raleigh JA: Reductive metabolism of the hypoxia marker pimonidazole is regulated by oxygen tension independent of the pyridine nucleotide redox state. Eur J Biochem 1998, 253:743-750.
25. Matsumoto S, Hyodo F, Subramanian S, Devasahayam N, Munasinghe J, Hyodo E, Gadisetti C, Cook JA, Mitchell JB, Krishna MC: Low-field paramagnetic resonance imaging of tumor oxygenation and glycolytic activity in mice. J Clin Invest 2008, 118:1965-1973.

26. Halestrap AP, Meredith D: The SLC16 gene family-from monocarboxylate transporters (MCTs) to aromatic amino acid transporters and beyond. Pflugers Arch 2004, 447:619-628.

27. Porporato PE, Dhup S, Dadhich RK, Copetti T, Sonveaux P: Anticancer targets in the glycolytic metabolism of tumors: a comprehensive review. Front Pharmacol 2011, 2:49.

28. Koch CJ: Measurement of absolute oxygen levels in cells and tissues using oxygen sensors and 2-nitroimidazole EF5. Methods Enzymol 2002, 352:3-31.

29. Epstein T, Xu L, Gillies RJ, Gatenby RA: Separation of metabolic supply and demand: aerobic glycolysis as a normal physiological response to fluctuating energetic demands in the membrane. Cancer Metab 2014, 2:7.

30. Garcia-Gonzalo FR, Cruz C, Munoz P, Mazurek S, Eigenbrodt E, Ventura F, Bartrons R, Rosa JL: Interaction between HERC1 and M2-type pyruvate kinase. FEBS Lett 2003, 539:78-84.

31. Cunningham J, Estrella V, Lloyd M, Gillies R, Frieden BR, Gatenby R: Intracellular electric field and $\mathrm{pH}$ optimize protein localization and movement. PLoS One 2012, 7:e36894.

32. Cuisnier O, Serduc R, Lavieille JP, Longuet M, Reyt E, Riva C: Chronic hypoxia protects against gamma-irradiation-induced apoptosis by inducing bcl-2 up-regulation and inhibiting mitochondrial translocation and conformational change of bax protein. Int J Oncol 2003, 23:1033-1041.

33. Graeber TG, Osmanian C, Jacks T, Housman DE, Koch CJ, Lowe SW, Giaccia AJ: Hypoxia-mediated selection of cells with diminished apoptotic potential in solid tumours. Nature 1996, 379:88-91.

\section{Submit your next manuscript to BioMed Central and take full advantage of:}

- Convenient online submission

- Thorough peer review

- No space constraints or color figure charges

- Immediate publication on acceptance

- Inclusion in PubMed, CAS, Scopus and Google Scholar

- Research which is freely available for redistribution 